\title{
Combined low-temperature scanning tumeling/atomic force microscope for atomic resolution imaging and site-specific force spectroscopy
}

\author{
Boris J. Albers, 1 Marcus Liebmann, 1,a) Todd C. Schwendemann,1 Mehmet Z. Baykara,1 \\ Markus Heyde,2,b) Miquel Salmeron,2 Eric I. Altman,3 and Udo D. Schwarz1,o). \\ 1 Department of Mechanical Engineering and Center for Research on Interface Structures and Phenomena, \\ Yale University, P.O. Box 208284, New Haven, Connecticut 06520-8284, USA \\ 2 Materials Science Division, Lawrence Berkeley National Laboratory, University of California, I Cyclotron \\ Road, Mailstop 66, Berkeley, California 94720, USA \\ 3 Department of Chemical Engineering and Center for Research on Interface Structures and Phenomena, \\ Yale University, P.O. Box 208286, New Haven, Connecticut 06520-8286, USA
}

\author{
a) Present address: II. Institute of Physics, Technical University of Aachen, \\ Aachen, Germany. \\ b) Present address: Fritz-Haber Institute of the Max-Planck Society, Berlin, \\ Germany. \\ c) Electronic mail: udo.schwarz@yale.edu.
}

We present the design and first results of a low-temperature, ultrahigh vacuum scanning probe microscope enabling atomic resolution imaging in both scanning tunneling microscopy (STM) and noncontact atomic force microscopy (NC-AFM) modes. A tuning-fork-based sensor provides flexibility in selecting probe tip materials, which can be either metallic or noumetallic. When choosing a conducting tip and sample, simultaneous STM/NC-AFM data acquisition is possible. Noticeable characteristics that distinguish this setup from similar systems providing simultaneous STM/NC-AFM capabilities are its combination of relative compactness (on-top bath cryostat needs no pit), in situ exchange of tip and sample at low temperatures, short turnaround times, modest helium consumption, and unrestricted access from dedicated flanges. The latter permits not only the optical surveillance of the tip during approach but also the direct deposition of molecules or atoms on either tip or sample while they remain cold. Atomic corrugations as low as $1 \mathrm{pm}$ could successfully be resolved. In addition, lateral drifts rates of below $15 \mathrm{pm} / \mathrm{h}$ allow long-term data acquisition series and the recording of site-specific spectroscopy maps. Results obtained on $\mathrm{Cu}(111)$ and graphite illustrate the microscope's performance.

\section{INTRODUCTION}

It is well known that scanning probe microscopes (SPMs) can be adapted to various environments such as air, liquids, or vacuum conditions and run at temperatures ranging from the millikelvin regime to hundreds of degrees celsius. This astonishing spectrum of possible operating conditions allows one to tailor the experimental setup to the specific scientific or technological problems that are to be addressed. Despite representing the most complex class of setups, SPMs working in ultrahigh vacuum (UHV) and at low temperatures (LTs) have become increasingly popular (see Ref. 1 for a recent review). This choice of operational conditions is necessary if effects manifesting only at low temperatures such as superconductivityz or the Kondo effect3 should be studied.

However, there are also two other reasons that make researchers develop low-temperature compatible setups. Firstly, low temperatures slow down or, in some cases, entirely suppress many processes that would otherwise be too fast or unstable to be observed, such as the surface diffusion and adsorption of atoms $4-6$ and molecules, 7,8 or surface reactions. 9 Secondly, if built appropriately, low-temperature SPMs can significantly reduce thermal drift, lower noise levels, diminish piezohysteresis, creep, and nonlinearities, and contribute to an enhanced stability of the atomic configuration of the tip apex.I Therefore, low temperature operation represents the preferred choice of researchers whenever ultrahigh resolution and long-term stability is of importance. Depending on the objectives, the design of low temperature SPMs can incorporate very different features. For the instrument presented in this paper, optimization for highresolution imaging in both scanning tunneling microscopy (STM) and noncontact atomic force 
microscopy (NC-AFM) modes was intended, as the system should ultimately enable atomic-scale imaging and site-specific, long-term spectroscopy of conducting and nonconducting surfaces with applications such as chemical imaging, the identification of catalytically active centers, atomic-scale growth studies, etc. To achieve this goal, excellent vibration isolation, a compact, rigid microscope design, superior temperature stability, and operation in ultrahigh vacuum were of primary concern. In addition, flexibility in the choice of tip materials (both metallic and nonmetallic) due to the application of a $Q$-plus style, tuning-fork-based sensor, 10,11 and the possibility to deposit molecules or atoms on the tip and/or sample while they are cold help provide control of the chemical nature of the tip-sample interactions. Finally, the use of an on-top bath cryostat allows not only in situ tip and sample exchange at low temperatures but also results in short turnaround times and budget-friendly cryogen consumption.

\section{SYSTEM OVERVIEW}

The UHV system is based on a commercial setup manufactured by Omicron Nanotechnology12 and consists of their standard preparation and analysis chambers in combination with a custom-made third chamber housing the microscope and the cryostat, referred to as "SPM chamber" (see Fig. 1 for an overview system drawing). Each chamber is equipped with an ion getter pump and a titanium sublimation pump. For bakeout and evacuation from ambient pressure, both the preparation chamber and the SPM chamber are additionally connected to rotary-vane-backed turbo pumps. To prevent the generation of unwanted vibrations, all mechanically operated pumps are turned off during measurements.

The preparation chamber currently features a sputter gun, a resistive heating stage, a plasma source, and a residual gas analyzer. In addition, it is connected to a separately pumped fast entry lock for introducing tips and samples into the vacuum system. Base pressures in the preparation chamber are typically in the low 10-10 mbar regime. In contrast, pressures below 10-11 mbar are routinely reached in the analysis chamber, i.e., lower than the calibrated range of the ion gauge used to measure it. At this time, the chamber is equipped with low-energy electron diffraction (LEED) and Auger capabilities (SpectaLEED combined LEED/Auger system from Omicron Nanotechnologyi2) and a second resistive heater stage.

The largest chamber of the system is the SPM chamber, which has been produced by NorCal (Ref. 13) according to our specifications. It was designed to have easy access to all parts of the microscope, which is cooled by an on-top bath cryostat (cf. Sec. III). Due to the chamber's large total volume and surface area caused by the cryostat, pressures in this chamber are typically in the upper 10-11 mbar regime. In addition, a sample carousel located in between all three chambers provides in-vacuum storage space for up to six tip or sample holders.

One of the features of this vacuum system is its gas inlet system. It allows dosing of molecules for adsorption at the tip apex and/or the sample surface both into the preparation and the SPM chamber, giving the experimentalists control over the chemical nature of the surfaces exposed during an experiment. Dosing is regulated by a total of four strategically positioned precision leak valves (type MDC ULV-150). 14 To avoid unnecessary chamber backfilling, stainless steel tubes have been fitted into the outlets of the leak valves attached to the SPM chamber to direct the molecules selectively either toward the tip or the sample, depending on which inlet has been chosen (see Fig. 1 for their locations). This permits working with the lowest amount of reagent gases possible. Deposition can be performed at room temperature as well as at low temperatures. Besides regular gases such as oxygen or carbon dioxide, the gas inlet system also accommodates liquids stored in glass containers (type MDC SEG-075).i4 Gas lines are pumped by a 3.21 / s rotary vane pump while the pressure is monitored by a convection gauge.

To achieve ultimate resolution, adequate vibration isolation is crucial. To meet the most stringent demands, the system has been set up in the basement of a building that was constructed with the requirements of highly vibrationsensitive equipment in mind. For example, the building has an extra thick base slab to minimize floor vibrations. Thereby, we purposely decided against having a separate foundation, as we believe that the high mass off the building (the weight of the base slab alone exceeds 1000 tons) has advantages compared to the necessarily lower weight of a separate foundation if not additional, potentially very expensive measures for vibration isolation are undertaken.is In addition, the system is located inside a soundproofed room. Operators, data acquisition electronics, as well as all other electronic components that might produce acoustic noise or need to be under surveillance are in a separate room during measurement. 
To further eliminate any noise sources, there are no outlets of the building's air handling system within the soundproofed room.16 Finally, the frame that supports the vacuum chambers is mounted on a total of 12 active vibration isolation dampers (type MOD-2 by Halcyonicsin) to minimize vibrations still introduced by floor movements.

\section{CRYOSTAT}

To cool the microscope, we chose an on-top bath cryostat fabricated by Cryovac.18 On-top bath cryostats have been rarely used for low-temperature AFM setups so far, 19,20 even though a number of such systems are in use for STM applications.21 The cryostat can store up to 8.51 of liquid helium (LHe) in its central dewar and $18 \mathrm{l}$ of liquid nitrogen (LN) in the nitrogen shield. With one filling of LHe, 75-96 h of measuring time are available, depending on whether or not the tuning fork preamplifier has been switched on (see Sec. VI). This results in a very low helium consumption of 2-3 1 per day.

The microscope is enclosed in a double set of thermal shields made of oxygen-free high-conductivity copper plated with $5 \mathrm{~m}$ of silver acting as an antimigration layer during bakeout followed by a $3 \mathrm{~m}$ thick layer of gold. The shields are connected to the helium (inner shield) and the nitrogen reservoir (outer shield), respectively (Fig. 2). As a consequence, the entire microscope is evenly cooled, suppressing the formation of thermal gradients within the microscope body. Both shields have a modular, square design.22 Each of the four side panels can be taken off independently to gain access to that side of the microscope, which makes servicing easy. Despite the double shields, we still have optical access to tip and sample at all times through infrared radiation blocking windows integrated into both side panels that are in line with viewports of the vacuum chamber. This simplifies the coarse approach, because the position of the tip over the sample can be monitored using a video camera positioned outside vacuum, which transmits live pictures to the operator's room.

By opening shutters that are integrated into the front shields, unrestricted access from dedicated flanges permits the direct deposition of molecules or atoms on either tip or sample. In addition, it also allows both tip and sample exchange using a wobble stick even while the microscope is cold. Samples cool in as little as $1.5-2 \mathrm{~h}$ to temperatures close enough to the final equilibrium value (typically $300-400 \mathrm{mK}$ off) that drift rates allow initial inspection, leading to short turnaround times. These values gradually stabilize further to less than $10 \mathrm{mK}$ off long-term equilibrium after $6 \mathrm{~h}$. The sample temperatures are measured with an $\mathrm{Si}$ diode23 located on the scanner directly underneath the sample holder and found to be about $5 \mathrm{~K}$ in STM mode and $6 \mathrm{~K}$ in AFM mode, as the tuning fork preamplifier produces some unavoidable heat (cf. Sec. VI). Temperature stability is currently about $1 \mathrm{mK} / \mathrm{h}$ in equilibrium, but could be improved further by either using a feedback loop-controlled heater located at the sample position and regulated by the adjacent temperature sensor, or by pumping on both the Lhe and the LN reservoirs. .4 Measurements would additionally benefit from the latter option, as it would decrease noise induced by the boiling of the LN and lower the temperature of the LHe (and thus of the microscope). Both approaches are possible in our setup, but have not been implemented yet.

All electrical signals enter or leave the chamber at the top of the cryostat. Most cables transmitting these signals are made from manganin (a copper-manganese-nickel alloy) and feature a wire diameter of only 0.1 $\mathrm{mm}$ to minimize heat transfer from room temperature to the microscope along the cables. Exceptions are the wires carrying the tuning fork signal (two wires), the tunneling current, the sample bias, and the oscillation excitation signal, which are coaxial cables made out of stainless steel. Most wires, each about 1 $\mathrm{m}$ long, are guided through five separate pipes passing through the He tank for precooling and electrical shielding. Once a wire leaves its pipe, it is thermally anchored to the He shields. At this point, the cable type is changed from the manganin wires described above to pure copper wires with diameters of $0.25 \mathrm{~mm}$. This facilitates the thermal stabilization of the microscope by optimizing the thermal conductance between shields and microscope provided by the wires.

\section{MICROSCOPE}

For the construction of the microscope, many of the well-tested design elements already employed in earlier LT-AFM developments two of the present authors have been involved in have been reused.20,25 The main innovation compared to these two earlier designs is the adoption of a tuning fork as a force sensor 
(see Sec. V), 10,11 as opposed to detection by fiber-based interferometry.26-28 This allows the realization of a more compact body, giving it dimensions simila to their sister STM versions of this design type (e.g., Ref. 29-32). For these microscopes, superior overall mechanical stability has been well established.32-39

Figure 3 depicts the microscope, whose main body is fabricated out of a single cylindrical Macor 40 piece 80 $\mathrm{mm}$ in height and $40 \mathrm{~mm}$ in diameter to ensure maximum rigidity. Most metallic parts are made out of titanium because its thermal expansion coefficient is similar to that of Macor.4l Additionally, the setup was designed to be as symmetric as possible around the scan axis to once again minimize thermal influence. In particular, like their predecessors, 20,25 it features a symmetry plane stretching from the front to the back, including the cylinder's middle axis (see the section view in Fig. 3). As a result, we could establish very low drift rates during measurement of $<2 \mathrm{pm} / \mathrm{h}$ vertically and $<15 \mathrm{pm} / \mathrm{h}$ laterally, the latter being equivalent to about one unit cell or less drift per day for typical sample materials. Initial drift rates shortly $(\approx 3 \mathrm{~h})$ after refilling cryogen or tip or sample exchange are about twice as high and need about one day to settle to the values given above. Since a clear correlation between the temperature stability and the position can be seen, we expect that these values reduce further once the temperature-stabilizing measures discussed in the previous section have been implemented.

In our setup, the sample is moved with respect to the tip. As before, 20,25 we continue to rely for the tipsample coarse approach on the "walker" principle, which has been introduced by Pan et al.30.31,42 This type of motor has been proven to operate very reliably and be mechanically stable at low temperatures in UHV. The piezostacks employed in this motor (termed as the $z$ motor later on) were prefabricated and supplied by PI Ceramic. 43 The sample is mounted on top of a piezotube scanner (type EBL 4 piezo ceramics, 44 length of $13.7 \mathrm{~mm}$, diameter of $9.5 \mathrm{~mm}$, wall thickness of $0.5 \mathrm{~mm}$ ) for scanning and $z$ feedback, which is embedded into the hexagonal sapphire prism that is moved up and down inside the $z$ motor. At room temperature, the scan range is about $1.6 \times 1.6 \mu \mathrm{m} 2$ in $x y$ and $\approx 300 \mathrm{~nm}$ in $z$ if driven by the $\pm 130 \mathrm{~V}$ provided by our highvoltage amplifier (cf. Sec. VI). These values decrease to $450 \mathrm{X} 450 \mathrm{~nm} 2$ and $110 \mathrm{~nm}$ in $x y$ and $z$, respectively, at LHe temperatures. A titanium rod, which is fixed at the bottom of the microscope, extends inside the scanner and acts as a stopper when the motor is fully retracted. With the scanner in this position, the rod also increases the scanner's mechanical stability during sample exchanges.

In typical measurements, the bias voltage is applied to the sample, while the tip is connected to the STM preamplifier (type RHK IVP 300) (Ref. 45) located outside the vacuum at the top of the cryostat. This configuration leads to an open-loop noise floor on experimental current data of $\approx 0.5 \mathrm{pA}$ peak-peak in a 500 $\mathrm{Hz}$ bandwidth. The microscope body is suspended by three springs from the helium tank base plate for vibration isolation from cryogen bubbling (cf. Fig. 2). An eddy current damping stage realized by mounting a copper cross below the Macor microscope body provides damping. In addition, a locking mechanism enables us to attach the microscope firmly to the helium shield for fast cool down as well as for rigidity during during tip and sample exchange.

\section{DEFLECTION SENSOR AND TIP HOLDER}

To date, the majority of LT-AFM systems in use are based on optical cantilever deflection detection systems such as fiber-optic interferometry (e.g., Refs. 19,20,25, and 46-51) or laser-beam deflection.52-5 These systems have the disadvantage that the integration of the deflection detection mechanism into the microscope design makes the microscope rather bulky. An additional disadvantage is that standard commercial cantilevers are used in these instruments, which limits the selection of materials available as tips. 57 To keep the microscopes compact and simple, some systems use piezoresistive cantilevers for measurement and signal detection, 58,59 which additionally eliminate the need of timeconsuming repositioning of the cantilever relative to the detection system upon probe tip exchange.

Despite these advantages, the resolution demonstrated by piezoresistive cantilevers at low temperatures has remained poor. Therefore, attention has turned toward other self-sensing devices, mainly ones based on quartz tuning forks. 50-64 Quartz tuning forks have been in use for SPM applications since 1989 (Ref. 65) and feature the same benefits of compact microscope design and positioning ease as piezoresistive cantilevers. However, they also offer a variety of additional merits that make them ideally suited to achieve many of our earlier formulated design goals: 
(1) It has been shown that choosing force constants typical for quartz tuning forks $(>1000 \mathrm{~N} / \mathrm{m}$ ) offers advantages for small amplitude imaging, which result in superior signal-to-noise ratios especially when short-range atomic interaction forces are to be probed.t. .

(2) 2) Quartz tuning forks allow mounting custom tips of considerable overall size (cf. inset in Fig. for an example), giving the experimentaist a wiảe choice of cifferent tip materials in their buik form. This is especially bencficial if the chemical interactions between certain specific atomic species should be explored.

(3) If conducting tip and sample materials are used, simultaneous operation in NC-AFM and STM modes is possible, $\ldots$ allowing force and tunneling current spectroscopy to be recorded at the same time.., Also, metallic STM-style tips can be shaped in situ by applying voltage pulses or by the controlled "gentle dipping" of the tip in the surface. Such procedures have been well developed in the STM community over the years, but cannot be applied to commercial siliconbased AFM tips.

As quality factor and signal-to-noise ratio were shown to increase significantly at low temperatures comparcd to room temperature operation, . low temperature operation of the tuning fork is even more favorable. Please note in this context also that quartz tuming forks do not produce significant amounts of heating power, in contrast to piezoresisitve cantilevers or detection systems based on laser light... Despite this impressive list of advantages, only two groups wave so far demonstrated atomic resolution at low temperatures using tuning forks (e.g., Refs. $5, .6$, and $7-7$. . Both their microscopes, however, do not offer the possibility of in situ tip exchange.

Our exchangeable tip holder is shown in Fig. It consist of a trapezoidal stainless steel metal plate $16 \mathrm{~mm}$ long, $7.6-11.0 \mathrm{~mm}$ wide, and $1 \mathrm{~mm}$ thick with a slit at the end so that it can be securely gripped by the wobble stick. Once it has been inserted into the microscope, the four springs indicated in Fig. enable electrical contact for the tuning fork and STM signals while providing mechanical stability at the same time. Note that in order to avoid any coupling between the STM signal, the tunneling current is collected using a thin wire contacting the tip separately (see inset in Fig. . The metal base plate of the tip holder is always grounded while in the microscope.

The tuning fork with an unmodified eigenfrequency of $32768 \mathrm{~Hz}$ is glued to a Macor piece in the so-called Q-plus configuration.. The prongs are both $2370 \mu \mathrm{m}$ long, $130 \mu \mathrm{m}$ wide, and $220 \mu \mathrm{m}$ thick, resulting in a force constant of $\approx 2000 \mathrm{~N} / \mathrm{m}$ (see appendix $A$ for details). The tuning fork is mechanically excited using a dither piezo. Resonance frequencies of up $1032 \mathrm{kHz}$ and quality factors ( $Q$ factors) of up to $50000 \mathrm{in}$ vacuum at low temperatures have been reached with tip attached (cf. Fig. S). Since Gildemeister et al. report $Q$ factors up to 150000 for even lower temperatures, we expect pumping on our cryostat will further increase the $Q$ factor.

For the resuits shown in this paper, tips were prepared from $25 \mathrm{\mu m} \mathbb{P t}$ Ir wire, which was glued to the end of the tuning fork prior to the tip sharpening process performed by electrochemical etching. The etching while the tip is in place has the additional advantage that the tip can be reetched and reused a couple of times before it needs to be replaced. To facilitate tip replacement, it is often possible to remove the tip wirc from the tuning fork and to glue a new wire on afterwards. However, this procedure cannot be repeated too often because the amount of glue and therefore the mass on the prong of the tuning fork increases with each tip replacement. We also cxperimented with other materials such as tungsten as tip material, but $\mathrm{Pt} / \mathrm{Ir}$ tips seemed overall the easiest to handle, most likely because of reduced ffects due to surfacc oxidation.

\section{W. ELECTRONIC SETUP}

As the electrical currents generated by the tuning fork oscillations are very weak, it is of preeminent importance that the distance between tuning fork and preamplifier is kept as short as possible. In our case, the two wires connecting the tuning fork with the preamplifier are about $10 \mathrm{~cm}$ long. The preamplifier itself is inside the vacuum and in contact with the LN shield. Having the feedback resistor at LN temperature reduces the intrinsic noise of the amplification stage, which is still able to work at this temperature without the nced to heat the operational amplifier (charges would freeze at LHe temperatures). On the downside. 
the temperature at the sample rises from $\approx 5$ to about $\approx 6 \mathrm{~K}$ with the amplifying stage switched on, and the measuring time that can be achieved with one filling of the helium bath is reduced by about $20 \mathrm{~h}$.

The preamplifier itself is a very simple current to voltage converter. Due to the high bandwidth and limited gain of the amplifier, a second amplification stage is necessary to achieve a convenient signal level. It is positioned outside the vacuum on top of the cryostat at a similar location as the STM amplifier described earlier. Keeping it outside provides easy access and allows to minimize the number of electronic components located within the vacuum chamber. Subsequently, the tuning fork signal passes a $35 \mathrm{kHz}$ lowpass filter to reduce high frequency noise picked up from sources such as, e.g., power supplies, etc. The $35 \mathrm{kHz}$ cutoff frequency was implemented as this choice does not have any impact on the frequency measurement, which naturally takes place at or below the $32 \mathrm{kHz}$ eigenfrequency of the tuning forks. For excitation, amplitude, and frequency control, a PLLPRO from RHK (Ref. 45) is used. Scanning, distance control, and data acquisition is carried out by the SPM 1000 system, also from RHK, in conjunction with their XPMPRO software package. This setup allows simultaneous recording of the topography (i.e., the $z$ voltage of the scan piezo), the frequency shift, the amplitude, the cantilever drive signal, as well as the tunneling current.

\section{FIRST EXPERIMENTAL RESULTS}

As suitable test samples, the (111) surface of copper was chosen for the STM mode and the (0001) surface of highly oriented pyrolytic graphite (HOPG) for the NC-AFM mode. This was not only because they are well known, $68,69,78-82$ but also because these surfaces challenge the system due to the low corrugation of their atomic structure in the respective modes.

\section{A. STM results obtained on copper}

Figure 6 shows topographical STM raw data acquired at $T=5 \mathrm{~K}$ on a $\mathrm{Cu}(111)$ single crystal, which had previously been cleaned by repetitive cycles of argon sputtering and annealing at temperatures up to $T=700$ ${ }^{\circ} \mathrm{C}$. The atomic lattice with a lattice spacing of $2.56 \AA$ is clearly visible. In addition, a nearby step edge just outside the imaged area in the upper left corner induces an electronic surface charge wave, which runs diagonally from upper left to lower right, but dies out after about half the image. Such surface charge waves are a well-known phenomenon on $\mathrm{Cu}(111)$ surfaces. 78,79 Note that stable STM imaging is possible even though the tip was mounted at the end of a tuning fork.

Another example for the same surface is shown in Fig. 7. Due to slightly different tunneling parameters and a different tip, the charge waves appear stronger relative to the atomic lattice than they did in Fig. 6 . Their specific appearance is due to interference of circular charge waves caused by the black defect in the upper half of the image and others originating from similar defects outside the present field of view. From the cross section displayed in Fig. 7(b), we see that the charge wave corrugation is of the order of $10 \mathrm{pm}$, i.e., similar as in Fig. 6, while the atomic corrugation is with 3-4 pm about ten times lower than before.

Generally, atomic corrugations as low as $1 \mathrm{pm}$ have been routinely resolved, thereby proving that the system is capable of operating as a fully functional STM without any compromises due to its additional AFM capabilities.

\section{B. NC-AFM results obtained on graphite}

Despite the good quality of the STM results shown above, we have to keep in mind that the main design goal for the instrument was to create an instrument capable of high resolution NC-AFM imaging. As mentioned earlier, we chose HOPG for the initial testing because it is easy to prepare, but difficult to image with NC-AFM due to weak tipsample interactions, which result in very low atomic corrugations. This is in sharp contrast to STM or contact mode AFM, where HOPG is a frequently used easy test sample. In fact, HOPG has so far been resolved in NC-AFM only by two other groups, both using high-resolution microscopes operated at helium temperatures. $68,69,80-82$

Figure 8 displays NC-AFM images of HOPG (raw data). Recorded at $T=6 \mathrm{~K}$ with oscillation amplitudes of $0.25 \mathrm{~nm}$, the images feature protrusions with $2.46 \AA$ spacing, which corresponds to the distance between 
every other atom in the graphite surface and agrees well with data recorded earlier under similar conditions. 80 Discussions why this structure-as opposed to the imaging of every atom in the graphite surface-is commonly observed in NC-AFM can be found in Ref. 81 and 82 . Corrugations were of the order of 4-5 pm [Fig. 8(a)] and 10-12 pm [Fig. 8(b)], respectively, with a noise level of below $1 \mathrm{pm}$ (cross section in Fig. 8(c)). Simultaneous to the topography data, the frequency shift (often termed as the "error signal," as $\Delta f$ is being kept constant), the excitation, the oscillation amplitude, and the tunneling current have been collected (not shown). The oscillation amplitude was calibrated using procedures described elsewhere. 83 Please note in this context that we usually did not detect a measurable tunneling current during the imaging of $\mathrm{HOPG}$ in $\mathrm{NC}-\mathrm{AFM}$ mode as the contact potentials were carefully compensated.

In the last two figures, force versus distance curves taken on HOPG are shown to demonstrate the instrument's usefulness for force spectroscopy. While Fig. 9 depicts an individual curve, 256 curves are combined in Fig. 10 to form a two-dimensional, site-specific array similar to 2D arrays described earlier.8486 From this figure, in which contour lines are drawn every $40 \mathrm{pN}$, we can see that local atomic-scale force variations can be observed up to a distance of about $0.15 \mathrm{~nm}$ from the level of closest approach to the surface, which has been arbitrarily gauged to zero. In both cases, the force was calculated from original frequency shift data applying a procedure introduced by Sader and Jarvis.87

\section{CONCLUSIONS}

We presented the design and first experimental results of a scanning probe microscope that can be operated in both STM and NC-AFM modes while operating in ultrahigh vacuum and at low temperatures.

Advantages of the design are excellent temperature stability, compact dimensions, low helium consumption, short turnaround times, provisions for in situ tip and sample exchange, and unrestricted access from dedicated flanges that allows optical access during operation and the deposition of molecules on tip and/or sample while they are cold. Initial experimental results show atomic resolution in both STM and NC-AFM modes with corrugations down to $1 \mathrm{pm}$ resolved and lateral drift rates as low as one unit cell per day.

\section{ACKNOWLEDGMENTS}

The authors thank R. D. Grober, A. Mugarza, and F. Ogletree for helpful discussions, J. Falter for help with some electronics, N. Pilet for assistance with data analysis, and S. Porthun and G. Lengel from RHK Technology for responding quickly to our wishes and suggestions while beta-testing their equipment and software. Financial support from the National Science Foundation (Grant No. MRSEC DMR 0520495), the Department of Energy's Office of Basic Energy Sciences (Catalysis and Chemical Transformations Program, Grant No. DoE DE-FG02-06ER15834), and the Petroleum Research Fund of the American Chemical Society (Grant No. PRF 42259-AC5) is gratefully acknowledged. The work at LBNL was supported by the DOE Office of Basic Energy Sciences, Materials Sciences Division, under Contract No. DE-AC02-05CH11231 and a personal stipend for M. H. from the Alexander von Humboldt Foundation.

\section{APPENDIX A: ESTIMATING THE SPRING CONSTANT OF THE TUNING FORK}

According to elementary elasticity theory, the spring constant of a cantilever can be calculated from $c z$ $=E t 3 w / 4 l 3$, where $E$ is Young's modulus, $t$ is the cantilever thickness, $w$ is its width, and $L$ is its length. With the values provided in the main text and Young's modulus as given further below, this results in $c_{z}=2056 \mathrm{~N} / \mathrm{m}$. However, as pointed out by Simon et al.,83 $c_{z}$ might change as the tuning fork is glued to its holder. Therefore, we simulated our setup (tuning fork and holder) by finite element analysis, which resulted in a $c_{z}$ of $1897 \mathrm{~N} / \mathrm{m}$. Thus, it seems adequate for calculations where the tuning fork's force constant is needed (e.g., when the force should be recovered from frequency shift data) to assume an average $c z$ of $\approx 2000 \mathrm{~N} / \mathrm{m}$. Please note that for the calculation of $c z$, we used a Young's modulus of $79.1 \mathrm{GPa}$, which is different from standard tabular values for quartz. This variation is caused by the tuning fork's special crystallographic cut of $\Theta=85^{\circ}$ and $\Phi=0^{\circ}$, which needs to be considered. 88 
Heidelberg, 2007)

2M. 'Tinkham, Introduction to Superconductivity (McGraw-Hill, New York, 1996).

3. Kondo, Thes, 32, 37 (1964).

4D. M. Eigler, P. S. Weiss, E. K. Schweizer, and N. D. Lang,

5P. S. Weiss and D. M. Eigler, Tre e. 69, 2240 (1992)

6D. M. Eigler and E. K. Schweizer, hrowerchom 344, 524 (1990).

7P. S. Weiss and D. M. Eigler, $71.3139(1993)$

PP. Zeppenfeld, C. P. Lutz, and D. M. Eigler, Ultramicroseopy $42-44,128$ (1992).

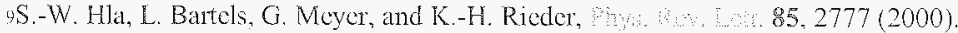

1)F. J. Giessibl, Appt. Rise C. 76, $1470(2000)$.

11F. J. Giessibl, S. Hembacher, M. Her\%, Ch. Schiller, and J. Mannhart, we whom I5, S79 (2004).

12Omicron NanoTechnology Gmbrf (www omicron.de), Limburger Strasse 75, 65232 Taunustein, Germany.

13Nor-Cal Products Inc. (www.n-c.com), 1967 So. Oregon, Yreka. CA 96097, USA.

14MDC Vacuum Products Corp. (www.mde-vacuum.com), 23842 Cabot Boulcvard, Hayward, CA 94545, USA.

15 Building vibrations caused by compressors, clevators, or air handling systems occur ai specific frequencies. If built correctly, a separate foundation can supress the transmission of these vibrations to the experimental setup. On the downside, the lower mass of a separate foundation makes it much more prone to external excitation by, e.g., traffic, nearby constnuction sites, seismic events, etc. Thus, while building peaks may be largely climinated from the vibrational noise spectrum, the overall noise floor of the spectrum is usually markedly higher compared to a spectrum taken on the building's base slab. As a consequence, if the microscope is built so that its resonant frequencies do not coincide with any building peaks, the low noise level offered by the building's base slab is better than that of a separate foundation. This is especially truc if in-house machinery can be prevented from exciting the building structure in the first place by, c.g., isolating them using simple rubber elements. To have the best of both worlds, some laboratories chose to additionally vibration isolate their separate foundations by dampers such as pneumatic springs. This setup is superior to both the solution in our laboratory and to the construction of a separate foundation, but costs much more. An architectural office specialized on the design of such facilities is, e.g., Wilson Architects, 374 Congress Strect, Suite 400, Boston, MA 02210. USA (http://ww. wilsonarch.com/index/nanotechnology)

If Scientists often request separate foundations when planning laboratory space for ultrahigh-resolution SPM work, but overlook the influence of acoustic noise from air landling systems on these measurements. Such systems are often yery loud, as building codes for laboratory space require substantial air recirculation and may thus cause significant disturbance of the measurements. In most cases, it is much cheaper to optimize the air handling system than to build a separate foundation; therefore, we suggest attacking this problem first. Optimally, close collaboration with the achitectural, engineering, and labor safety oflices involved in the planning and construction process should result in a solution that avoids any air outlet inside the soundproofed room that might potentially produce audiable noise duing measurement. Even if no separate soundproofed room is installed, planning specifically for low-noise air handling might still be an effective measure to increase stability and resolution.

17 Halcyonics GmbH (www halcyonics.de), Tuchmacherweg 12, 37079 Göttingen, Germany.

1k Cryovac Gesellschaft fur Tieftemperaturtechnik mbH \& Co KG (www.cryovac.de), Heuscrweg 14, 53842 Troisdorl: Germany.

14.Y. Suehira, Y. Tomiyoshi. Y. Sugawara, and S. Morita, Re.. 72,2971 (2001).

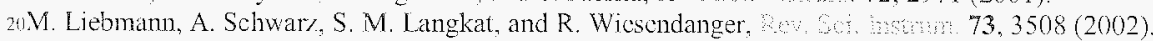

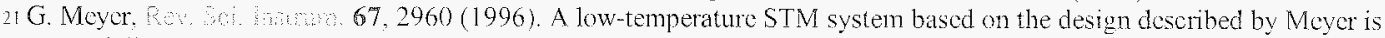
commercially available by VTS-CreaTec GmbH (www.vts-creatcc.com), Industricstr. 9, 74391 Erligheim, Germany. In addition, at somewhat similar system is offered by Omicron Nanotechnology (Ref. 2 )

22 Modular double-shield cryostat systems have been described earlier for STM applications in connection with flow cryostats; sec, B. C. Stipe, M. A. Rezaci, and W. Ho, Ses, Se. Her. 70, 137 (1999), and A. M. Lakhani, S. J. Kelly, and T. P. Pcarl, hil, 77. 043709 (2006)

23Type DT-470-SD made by LakeShore Crotronics, Inc. (www lakeshore-com), 575 McCorkle Blvd, Westerville, OH, 43082, LSA. 24The boiling temperause of the cryogens, and thus the temperature of the cryostat and ultimately the entire microscope, depends on ambient pressure if the cryogens are cxposed to air. In fact, the boiling temperature of LHe ehanges about $1 \mathrm{mK}$ for 1 mbar change in atmospheric pressure (according to data published on www.aidiquide. Com), which is so signilicalnt that we suspect this to be the most important factor currently limiting our temperature stability. Note that the boiling temperature of L.N shifts even more. namely, $\approx 7.5 \mathrm{mK} / \mathrm{mbar}$. As discussed in the main text, we plan on improving temperature stability in the future by pumping on both LHe and LN.

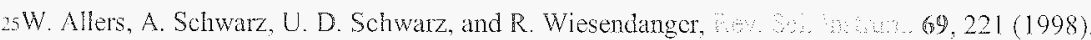

26 D. Rugar, H. I. Marmin, R. Erlandsson, I. E. Stern, and B. D. Terris, $23 \%$, 2337 (1988).

27 D. Rugar, H. Mamin, and P. Gucthner, Af. 55,2588 (1989)

28 A. Moser, H. J. Hug, Th. Jung, U. D. Schwarz, and H.-J. Güntherodt, Pas a, 769 (1993).

29) S. H. Pan, S. Behler, M. Bemasconi, and H. J. Gïntherodt, Bull. Am. Phys. Soe. 37, 167 (1992).

3) Chr. Wittneven, R. Dombrowski, S. H. Pan, and R. Wiesendanger, ...

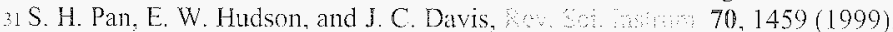

32 I. Wiebe, A. Wachowiak, F. Meier, D. Haude, T. Foster, M. Morgenstern, and R. Wiesendanger,

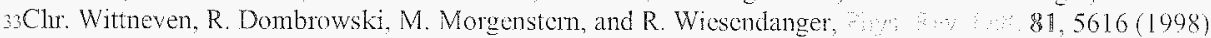

${ }_{34}$ E. W. Hudson, S. H. Pan, A. K. Gupta, K.-W. Ng, and J. C. Davis, 285, 88 (1999).

${ }_{35}$ S. H. Pan, E. W. Hudson, K. M. Lang, H. Eisaki, S. Uchida, and J. C. Davis, $1403,746(2000)$

36 M. Morgenstem, Chr. Wittneven, R. Dombrowski, and R. Wiesendanger, $18.8588(2000)$.

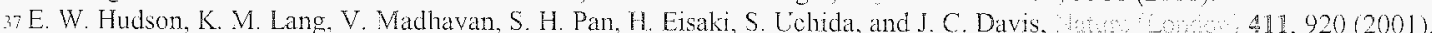

38. J. E. Hoffinan, K. McElroy, D.-H. Lee, K. M. Lang, H. Eisaki, S. Uchida, and J. C. Davis, 51729297.1148 (2002).

3) M. Morgenstem, J. Klijn. Chr. Meyer, M. Getzlaff, R. Adelung, R. A. Römer, K. Rossnagel, L. Kipp, M. Skibowski. and R.

Wiesendanger, the $89,136806(2002)$.

4)MACOR is a trademark of Coming Glass Works and denotes a machinable glass ceramic.

4 G. Nuncs and D. Williams, 100 . 13,1063 (1995).

42 S. H. Pan, International Patent Publication No. WO 93/19494 (30 September 1993). 


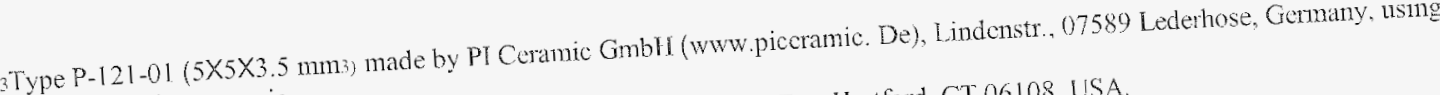
Prestige Park Circle, East Hartord CT 06108. USA

FBL Products Ine. (www eblproduets com),

4 RHK Technology (www rhk-tch com), 1050 Last Maple Road, Troy, M7 48083).

${ }_{46}$ R. Euler, U. Memmert, and U Marmant, Jo, 70,14 (1999)

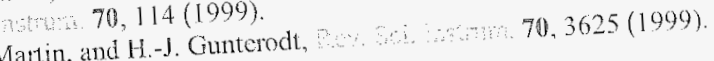

4. H. J. Hug, B. Stiefel, P. J. A. van Schendd, A. Moster, S. Mant

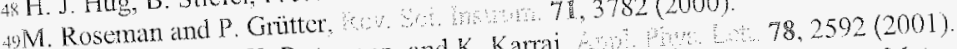

the Retersson, and K Kartal,

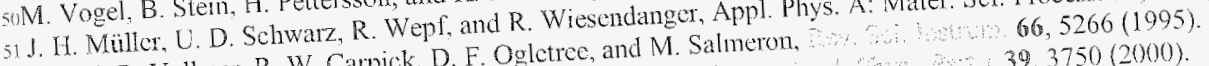

5 Dai R. Vollmer, R. W. Carpick, D. F. Ogletree, and M. Saheron, $\quad 39,3750(2000)$

氵3 K. Suruki, M. Iwatsuki, S.-I. Kilamura, and Ch. B. Mooncy, 051106 (2007)

${ }_{4}$ C Garza. L. T. Thieghi, and R. Castillo, $126,051100(2007)$ rom temperature are offored commercially by 5-AFMs based on laser-beam deflection that can be op

Omicron (Ref. O), RHK (Ref A), and Jeol (Ref S). Akishma, Tokyo 196-8558, Japan.

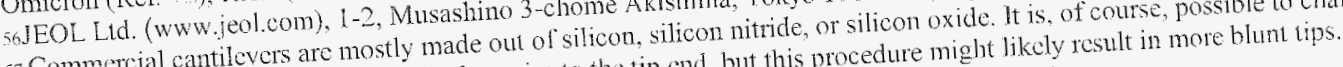

57 Commercial cantilevers are mosty made out of sticut, sildeon but this procedure might likcly tos

material by evaporating another chenical specie

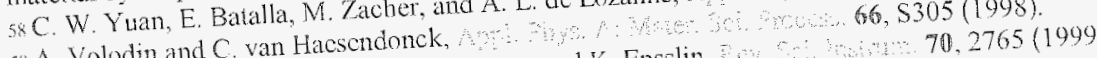

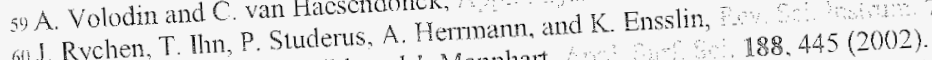

S. Riessibl, and I Mannhart,

$188,445(2002)$
$0,75,2446(2004)$

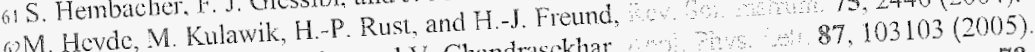

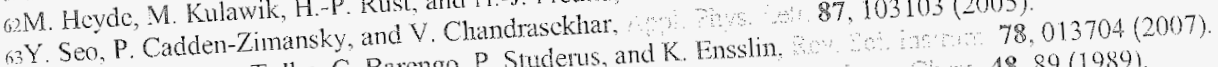

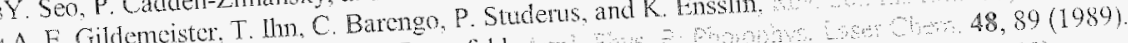

6 A. E. Gidemcister. T. 1hom, and K. Dransfeld, $40,352(1999)$

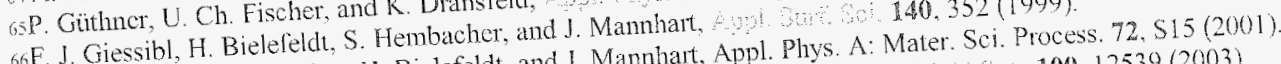

66 F. J. Giessibl, H. Bielefeld. S. Helcfeldt, and I. Mamhat, Appl. Phys. A. Mater. Sc1. $1009.12539(2003)$

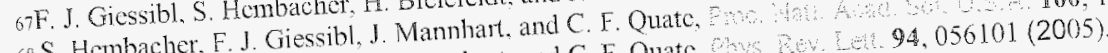

ia S. Hembacher, F. J. Giessibl, Mannhart and C. F. Quate,

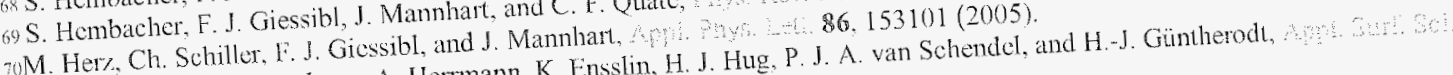

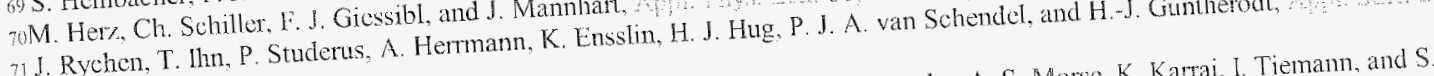

$157,290(2000)$

72 R. D. Grober, J. Acimovic, I. Schuck, D.

, Ens van Schendel, and H.-J. Günthcrodt.

j. Rycher, $T$ Thn P. Studerus. A. Hemman

73.J. Rychen, T. Thn, P. Studerts. A. \%) shows on their corporate website as their "result of the month $11 / 2006$ "atomic resolution
$71,1695(2000)$. a shows on their corporate webite as their "result of these results have not becn published images of $\mathrm{NaCl}(001)$ obtained with a $Q$-plus style sensor at low tetupuratures. However,

otherwisc.
.

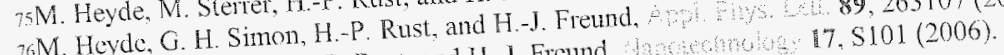

M. Hevie, M. Sterrer, M.-P. Rust, and H.-J. Fround, Thenemb $26218(1993)$

77 M. Heyce, M. Ste L. Lutz, and D. M. Eigler, 369,464 (1994).

$7 \mathrm{M}$. F. Crommic, C. P. Lum, and C. P. Luly, and D. M. Eigler, 369,464 (1994).

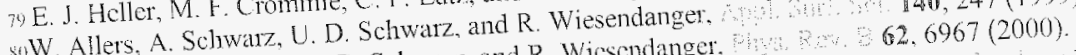

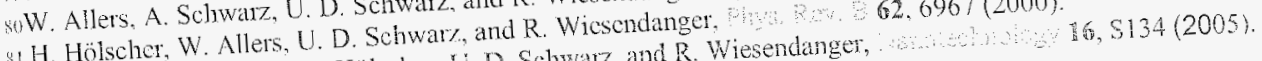

$\$ 1 \mathrm{H}$. Hölscher, W. Allers, U. D. Schwar, 2 . D. Schwarz, and R. Wiesendanger,

M. Ashino, A Schwar, H. Holscher, C. D. Schwar, and R. W 255503 (2007).

s. G. H. Simon, M. Heyde, and H.-P. Rust, and R. Wiescndanger, $8.4428(2002)$.

H. Holscher, S. M. Langkat, A. Schwaz, and R. Wlescndanger, 527, 12 (2003).

s. M. Langkat, M. Hökcher, A. Schwarz, and R. Wesendang, 136101 (2006).

a Schimeisen, D. Weiner, and $\mathrm{H}$. Fuchs, $841801(2004)$

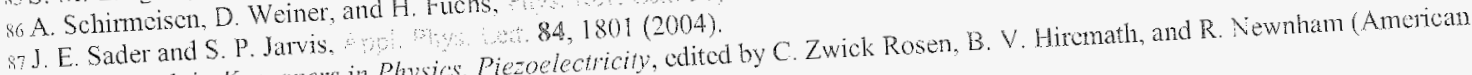

88 R. W. Ward, in Kevpopers in Phystu. Ples

unstitute of Physics, New York, 1992), p. 220 


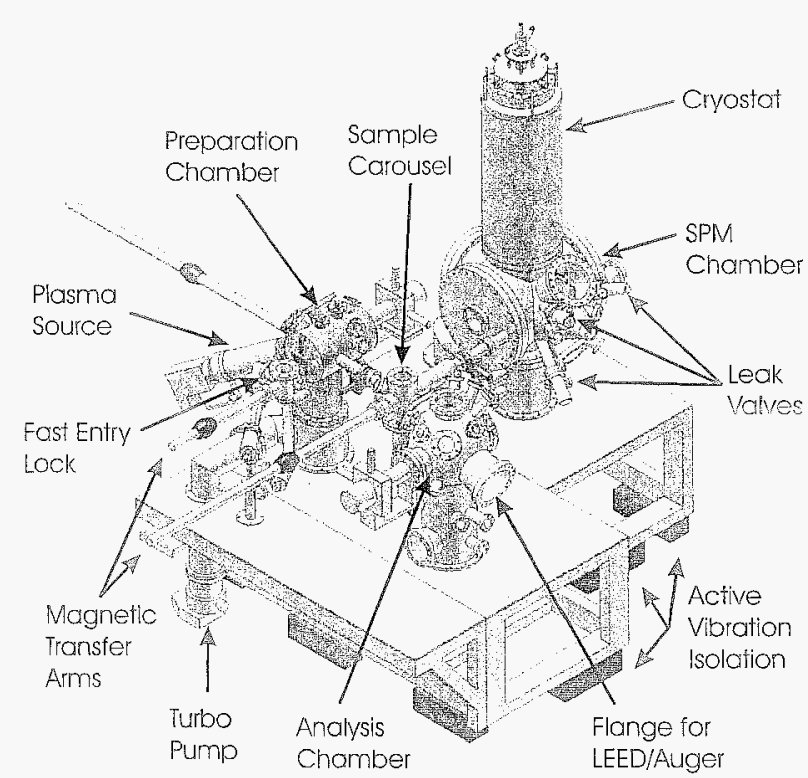

FlG. 1. Overvicw of the vacuum system including cryostat. Please note that most flanges are drawn open for clarity, i.e., without viewports, feedthroughs, or small equinment such as LEED/Auger, etc., in place

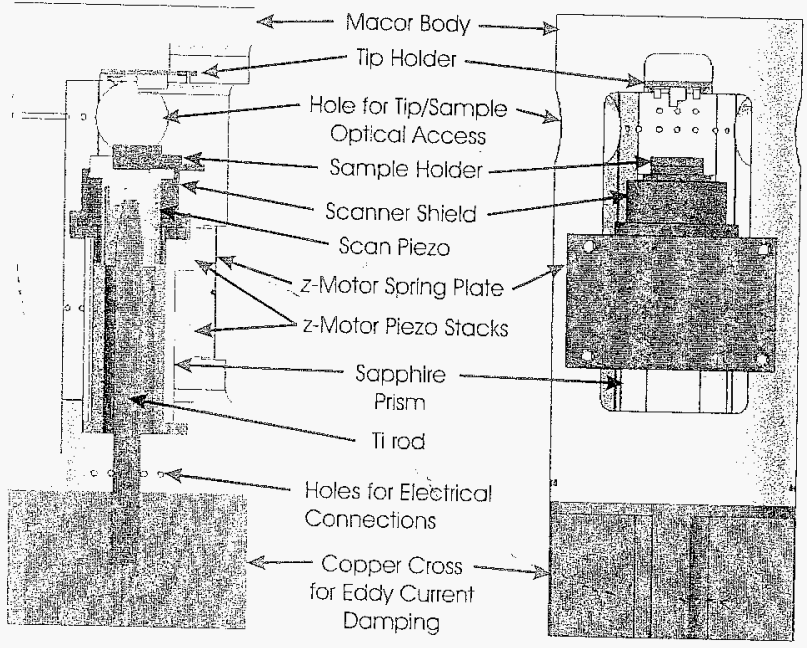

FIG. 3. (Color online) Section view (left; cut along the symmetry plane) and front view (right) of the microscope. For details, see text.

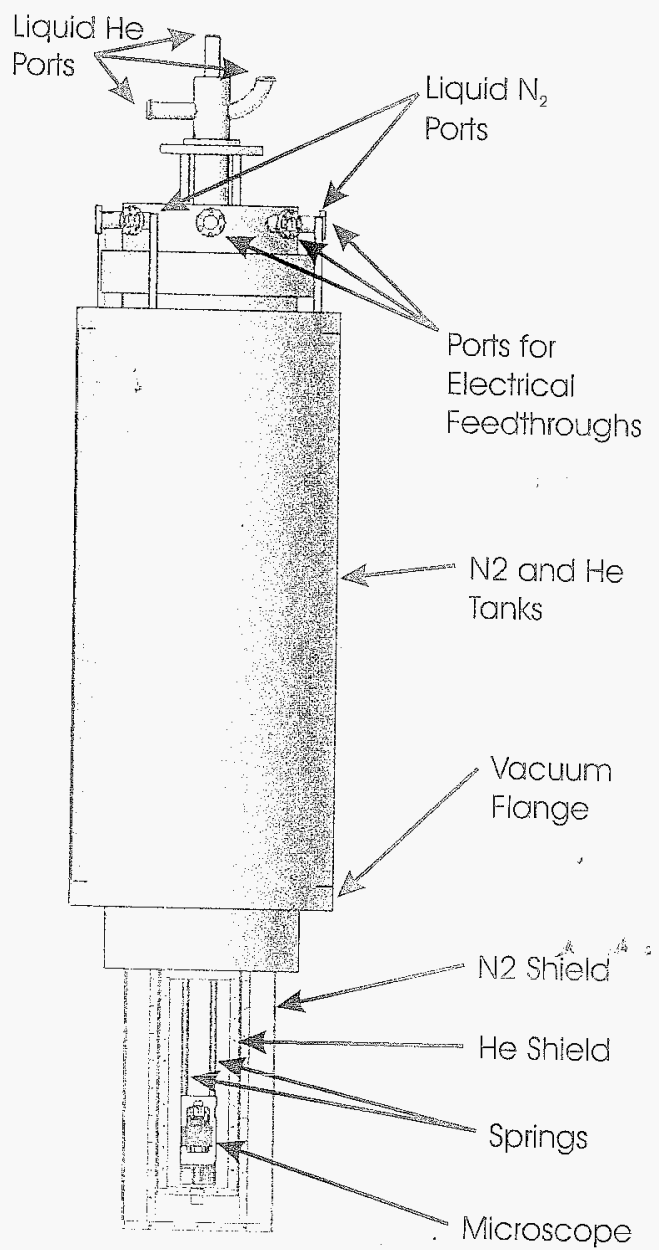

FIG. 2. (Color online) Cryostat with squared $N_{2}$ and He shields. For better visibility, the front shields are removed, exposing the microscope hanging on springs.

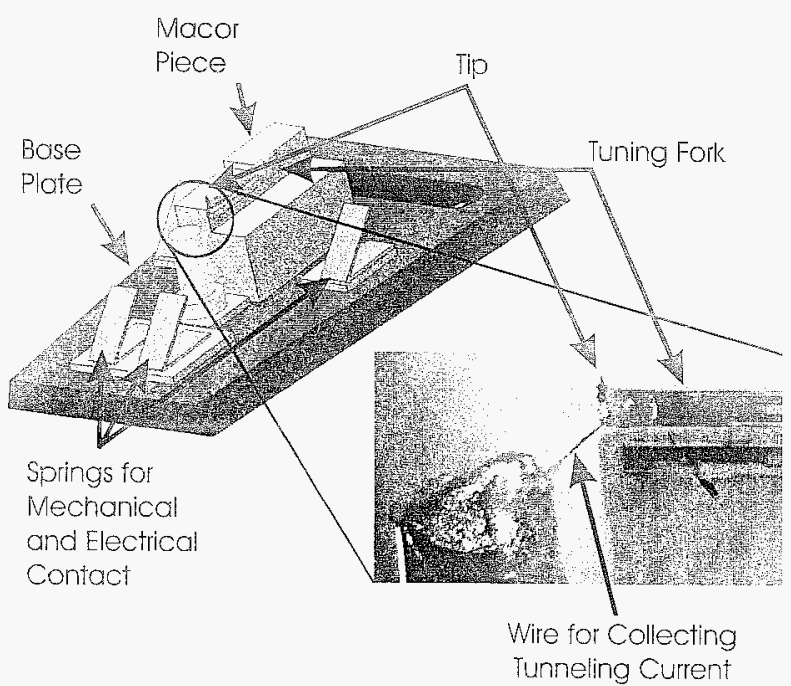

FIG. 4. (Color online) Sketch of the replaceable tip holder with tuning fork and custom tip. Electrical comections from the springs to the tuning fork and tip. respectively, are omitted for clarity. Inset: photo of tip, tuning fork and $57 \mathrm{M}$ connection. The wire for collecting the tumeling current has a dianceter of $25 \mu \mathrm{m}$. 


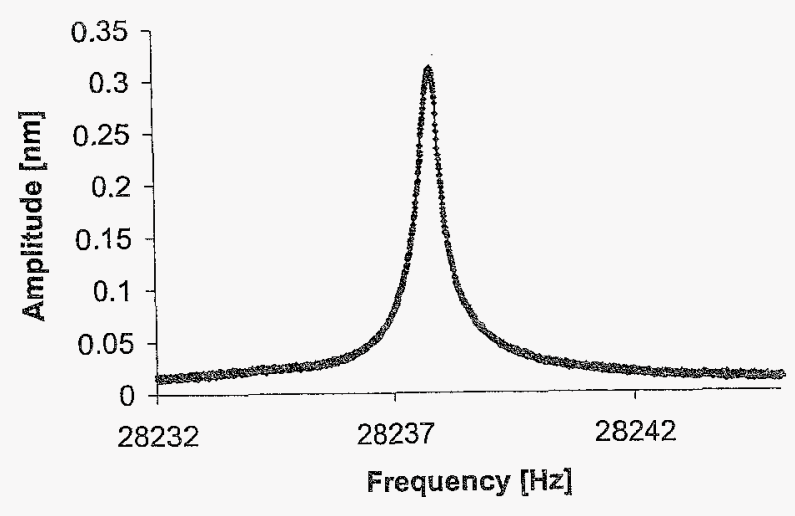

G. 5. (Color online) Example of a resonance curve of a tuning fork with / Ir tip attached. The data have been acquired in vacuum at $T=6 \mathrm{~K}$, aturing a resonance frequency of $f_{0}=28237.8 \mathrm{~Hz}$ and a $Q$ factor of $=39200$.

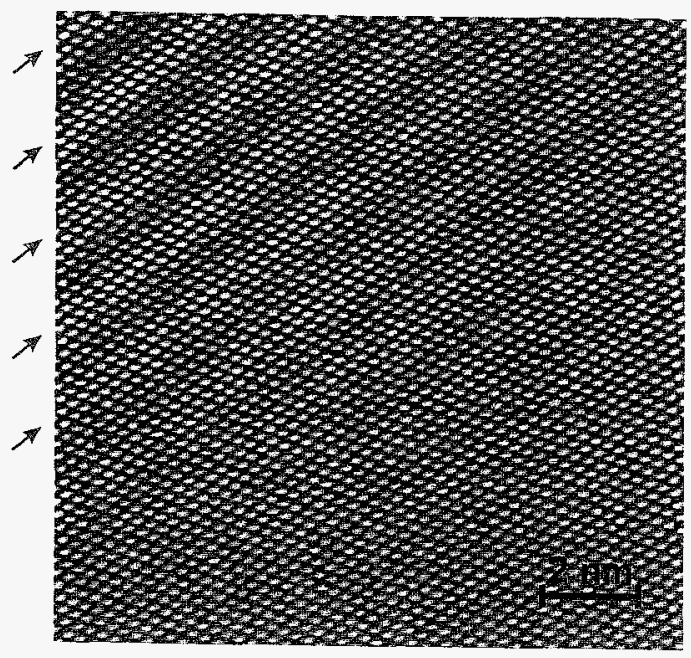

FIG. 6. (Color online) STM image (raw data) of $\mathrm{Cu}(111) \quad(I=50 \mathrm{pA}$ $U=+100 \mathrm{mV}, T=5 \mathrm{~K}$, scan speed of $13 \mathrm{~nm} / \mathrm{s}$, image size: $13 \times 13 \mathrm{~nm}^{2}$ ) An electronic surface charge wave, caused by a step edge located just outside the scanned area next to the upper left corner, rúns diagonally from upper left to lower right. The arrows on the left indicate the position and orientation of the individual wave fronts. Atomic corrugation is $\approx 30 \mathrm{pm}$ and charge wave corrugation $\approx 10 \mathrm{pm}$ max.
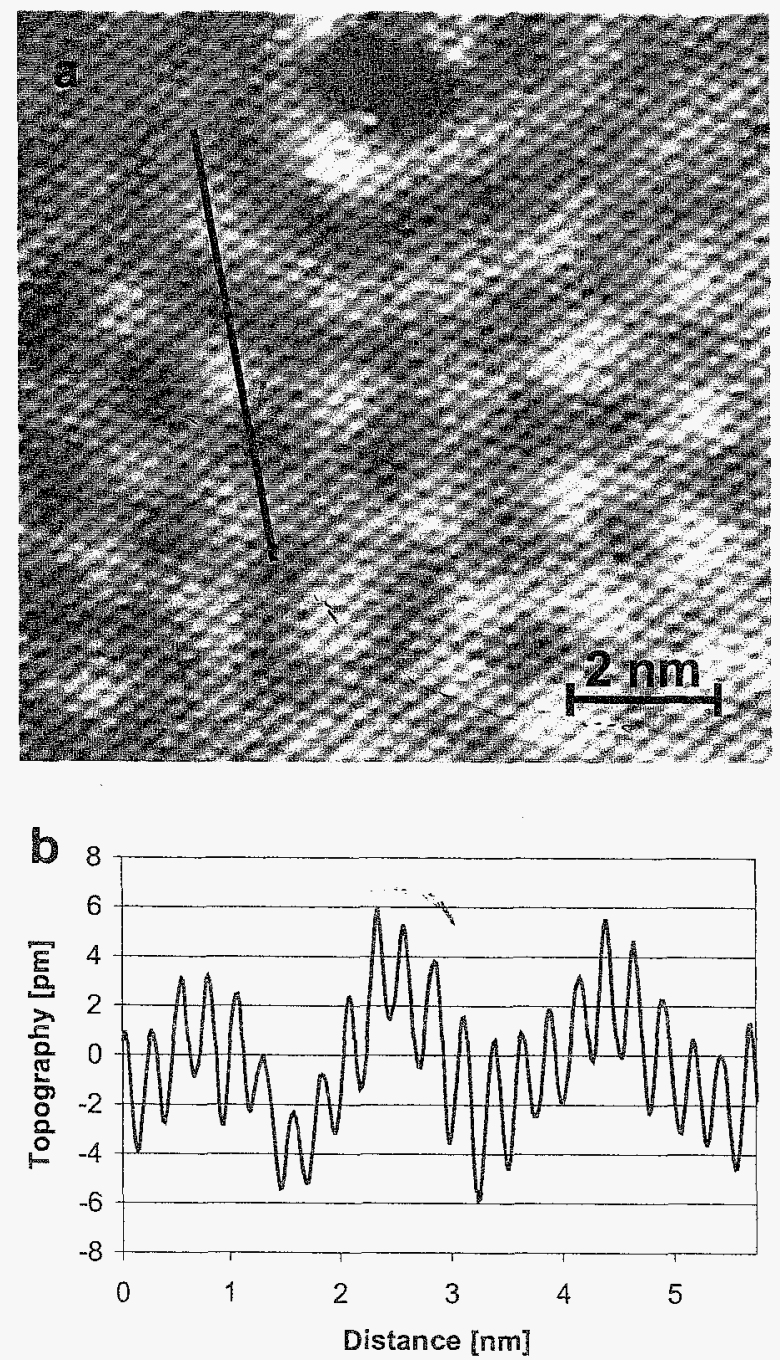

FIG. 7. (Color online) (a) STM image (image size: $10 \times 10 \mathrm{~nm}^{2}$ ) recorded on $\mathrm{Cu}(111)$ at $T=5 \mathrm{~K}$ with a tunneling current of $I=300 \mathrm{pA}$, an applied bias voltage of $U=+20 \mathrm{mV}$, and a scan speed of $11.1 \mathrm{~mm} / \mathrm{s}$. Both the atomic surface comugation as well as the charge modulations originating from several interfering circular charge waves can be observed. The data shown have been slightly Fourier filtered. (b) Section view along the line shown in (a). 

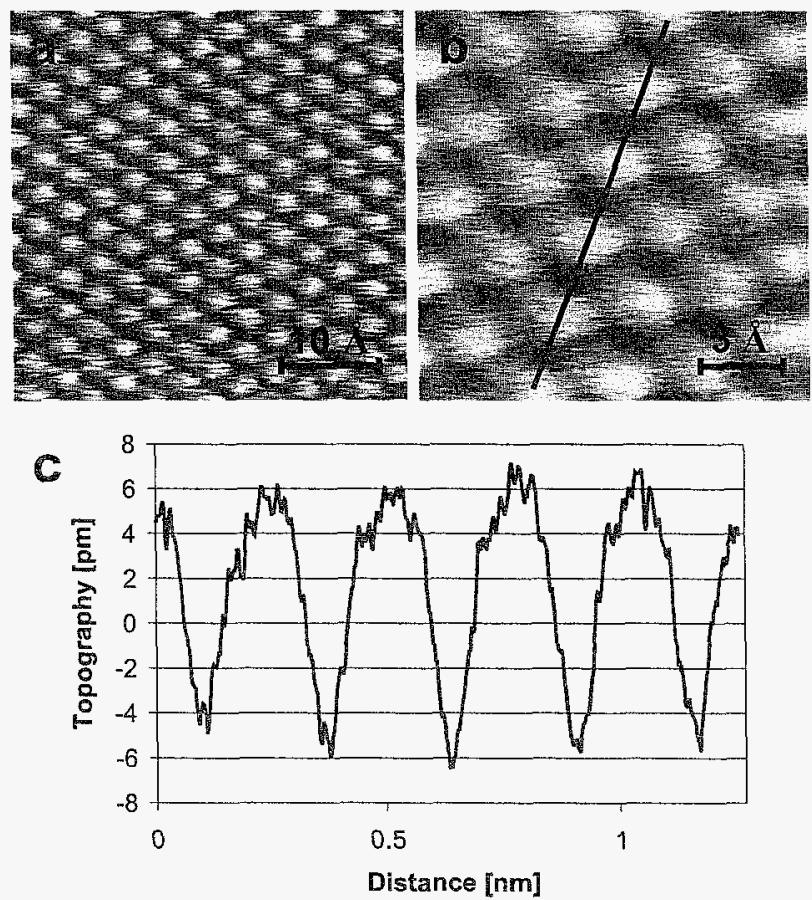

FIG. 8. (Color online) [(a) and (b)] Atomically resolved NC-AFM images of HOPG, recorded with constant oscillation amplitudes of $0.25 \mathrm{~nm}$ and constant scan speeds of $1.3 \mathrm{~nm} / \mathrm{s}$ using a tuning fork with $f_{0}=29529 \mathrm{~Hz}$. The sample has been cleaved in air and subsequently been heated in vacuum to $150{ }^{\circ} \mathrm{C}$ for $30 \mathrm{~min}$ to remove eventual contamination. The images were recorded at $\Delta f=-3.1 \mathrm{~Hz}$ with the PLL bandwidth limited to $18.75 \mathrm{~Hz}$ for (a) and at $\Delta f=-2.9 \mathrm{~Hz}$ with $37.5 \mathrm{~Hz}$ PLL bandwidth for (b); image sizes are $3.8 \times 3.8 \mathrm{~nm}^{2}$ and $1.3 \times 1.3 \mathrm{~nm}^{2}$, respectively. (c) Section vicw cut along the line indicated in (b). All data shown are raw data.

Rev. Sci. Instrum. 79, 033704 (2008)

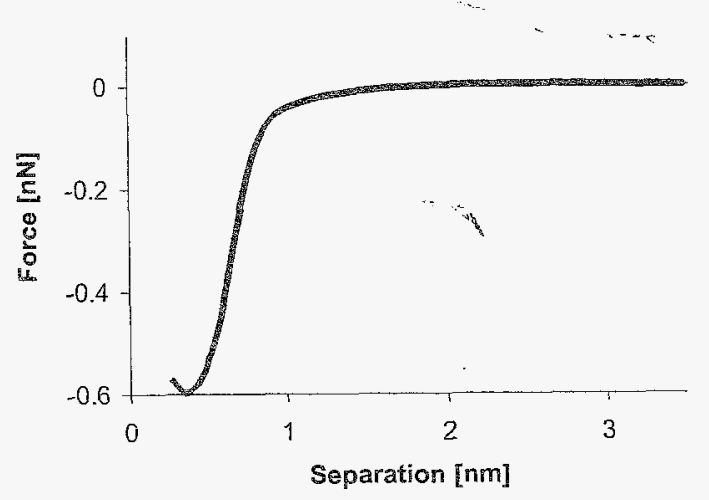

FIG. 9. (Color online) Force curve obtained on HOPG; oscillation amplitude was $0.23 \mathrm{~nm}$. Note that despite the usage of an etched Pt/Ir tip as probe, long-range forces are comparatively small.

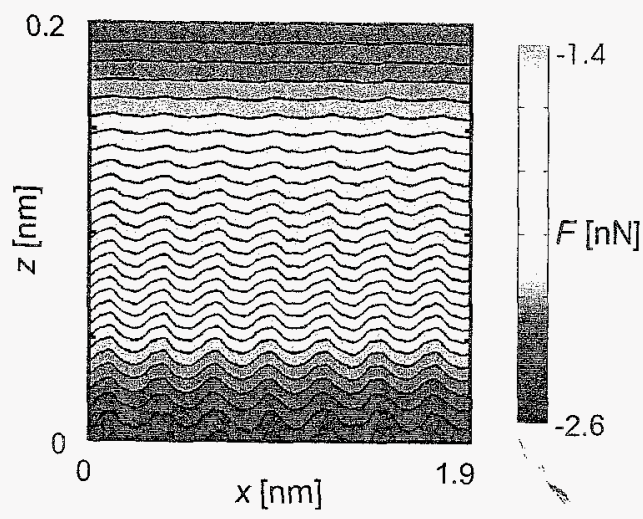

FIG. 10. (Color online) Two-dimensional array of 256 force curves recorded along a line of $1.9 \mathrm{~nm}$ length on HOPG. Contour lines of equal force, plotted every $40 \mathrm{pN}$, visualize force variations that are induced by the atomic lattice of the graphite sample. Note that atomic corrugation can be observed up to a height of $\approx 0.15 \mathrm{~nm}$ above the distance of closest approach. 